\title{
Mobilité des individus et familiarité construite : des arrangements qui offrent aux touristes des prises pour parcourir le monde
}

Philippe Violier

\section{(2) OpenEdition}

Journals

Édition électronique

URL : http://journals.openedition.org/tourisme/1365

DOI : 10.4000/tourisme.1365

ISSN : 2492-7503

Éditeur

Éditions touristiques européennes

Référence électronique

Philippe Violier, «Mobilité des individus et familiarité construite : des arrangements qui offrent aux touristes des prises pour parcourir le monde », Mondes du Tourisme [En ligne], 12 | 2016, mis en ligne le 01 décembre 2016, consulté le 03 mai 2019. URL : http://journals.openedition.org/tourisme/1365 ; DOI : 10.4000/tourisme.1365

Ce document a été généré automatiquement le 3 mai 2019.

\section{c) $(1) \ominus$}

Mondes du tourisme est mis à disposition selon les termes de la licence Creative Commons Attribution - Pas d'Utilisation Commerciale - Pas de Modification 4.0 International. 


\title{
Mobilité des individus et familiarité construite : des arrangements qui offrent aux touristes des prises pour parcourir le monde
}

\author{
Philippe Violier
}

1 Il existe deux manières idéal-typiques d'habiter le monde, le régime sédentaire et la mobilité, qui peuvent se combiner selon des arrangements variables en fonction des individus et des groupes. Cette dernière impose aux individus la confrontation à l'altérité définie comme « la qualité de ce qui est autre pour un individu dont l'identité personnelle («identité je ») et l'identité sociale («identité nous ») sont les référents familiers, voire non questionnés par rapport à un monde étranger dont les normes et conventions et manières de faire sont radicalement autres » (Stock, 2008, p. 147). La compréhension de la capacité des individus à affronter la diversité du monde fait l'objet de trois démarches scientifiques. L'une met l'accent sur le capital spatial et explore les savoirs et savoir-faire qui permettent aux individus d'affronter cette altérité de manière inégale, puisque ce capital est à la fois individuel et différencié socialement (Lévy, 2013). La seconde s'intéresse aux médiations établies entre les mondes, depuis les voyagistes jusqu'à internet, et qui facilitent la circulation (Ceriani, Coëffé, Gay et alii, 2008). Mathis Stock rassemble ces deux analyses dans un continuum entre prise en charge et autonomie fondée sur des compétences acquises par les touristes (Stock, 2008). Une dernière approche abordera au contraire les actions conduites dans les lieux des mondes et qui visent à en faciliter l'accès. Elle mettra donc l'accent sur l'interaction sociale et spatiale puisqu'elle étudie comment les acteurs dans des espaces, étudiés du point de vue de l'accueil, fabriquent de la familiarité, qui en réduit l'altérité objective ou y facilite pour les individus mobiles la confrontation avec l'altérité. Notre article vise à appréhender de manière théorique et pragmatique cette construction et contribue à affiner l'analyse des technologies spatiales, soit les «éléments de médiation [...] qui vise[nt] à résoudre des problèmes d'espace $[. .$.$] distance, [. .$.$] accès, [...] altérité » (Stock, 2008, p. 157).$ 
2 La pratique touristique est affectée comme d'autres institutions par l'autonomisation croissante des individus et le déclin corrélatif des pratiques sociales très structurées par les organisations, les tour-opérateurs notamment, qui ont permis au plus grand nombre de s'affranchir, dès la seconde moitié du XIX ${ }^{\mathrm{e}}$ siècle, des contraintes imposées par l'altérité (Tissot, 2000). Ainsi en 2014, selon la DGCIS ${ }^{1}$, seulement 15,4 \% des personnes qui résident en France ont recours à un service tout compris pour mettre en œuvre leur pratique touristique. Cette part, qui n'atteint que 7,8 \% lorsque les personnes restent en France, passe cependant à $39,5 \%$ dès lors qu'une frontière est franchie. Cette autonomisation des individus touristes accroît l'enjeu pour les acteurs territorialisés et engagés dans le développement local de construire de la familiarité. En effet, si les touristes souhaitent s'affranchir de l'encadrement, il importe que l'expérience se passe sans dommage afin que le discours produit au retour soit, du point de vue des acteurs de la destination, incitatif pour d'autres individus.

3 Cependant, la pratique touristique ne met en jeu les différentiels d'altérité les plus marqués qu'à la marge. En effet seule une minorité des touristes est réellement confrontée à des degrés élevés d'altérité. La plupart des mobilités relevant du tourisme dit international, marquées par un franchissement de frontière selon la définition promue par l'Organisation mondiale du tourisme, se limitent aux pays proches, et dans de nombreux États le tourisme intérieur est davantage pratiqué. Dans la plupart des États européens, par exemple, les Européens constituent 85 à $90 \%$ des touristes non résidents, y compris parmi les pays les plus visités ${ }^{2}$ : France $87 \%$, Espagne $93 \%$, Italie $87 \%$. Inversement, les villes constituent des espaces de médiation du rapport au monde. La plupart des touristes sont des citadins qui ont de l'urbain une expérience disponible pour être réinvestie dans la pratique touristique. Nous remarquons ainsi que les seules possibilités de circulation autonome dans les espaces de forte altérité ont longtemps été les villes; par exemple, si la plupart des tour-opérateurs ne proposent que des circuits accompagnés en Chine, il est possible de séjourner de manière autonome, en réservant seulement le vol et l'hébergement, dans les métropoles de Beijing ou de Shanghai.

Nous cherchons donc à appréhender la familiarité construite. Dans une première partie nous approfondirons la question de l'altérité et nous la déclinerons notamment de manière concrète, telle qu'elle se pose aux individus engagés dans une mobilité touristique. Ensuite nous montrerons de manière pragmatique comment l'altérité réelle peut-être compensée par une familiarité construite et sur quels ressorts repose cette dynamique. Cette démarche est fondée sur une observation participante mise en œuvre dans les réseaux de métro de métropoles situées dans différents mondes. Enfin nous en déduirons que l'interaction sociale à travers le tourisme contribue à rendre le monde habitable.

\section{Les touristes aux prises avec l'altérité}

Le tourisme est une mobilité qui a pour finalité la recréation des individus par des pratiques de repos, de jeu ou de découverte (Équipe Mit, 2002 ; Knafou et Stock, 2003 ; Stock, 2003). Le repos se conjugue avec la familiarité (Stock, 2001 ; Équipe Mit, 2002). Mais cette dernière peut être largement construite, si bien que, même dans une situation de forte altérité objective, les touristes peuvent se percevoir en situation de grande familiarité dans un établissement correspondant aux normes sociales (cuisine internationale, personnel multilingue, retranchement garantissant l'entre-soi et la 
sécurité...). Le comptoir est ainsi l'outil privilégié de la diffusion du tourisme dans le monde (Gay, 2000). C'est un type de lieu créé ex nihilo, fermé, maîtrisé par un opérateur (Équipe Mit, 2002; Stock, 2003). Dans cette situation les touristes peuvent jouir des aspects vécus comme positifs de l'altérité bioclimatique (le soleil par exemple pendant l'hiver des zones tempérées) sans en affronter les modalités plus contraignantes (la chaleur excessive de la nuit, les agressions des insectes ou les risques des maladies endémiques) ni se confronter à l'altérité sociale. De même, dans les représentations occidentales, le repos est associé aux bords de mers depuis l'invention du littoral (Corbin, 1990 ; puis Knafou, 2002), et chaudes depuis que les Américains ont ouvert la voie des côtes ensoleillées (Équipe Mit, 2005). Or, à côté des comptoirs, les stations ont été les lieux de cette conquête, des lieux construits pour le tourisme, qui sont donc en euxmêmes des formes de médiation spatiale facilitant la territorialisation du touriste (pour la typologie des lieux touristiques voir Équipe Mit, 2002 ; Stock, 2003). Dans les stations les touristes se tiennent, à l'origine du moins, à l'écart des populations locales, et l'organisation spatiale, structurée par rapport à la mer ou à la montagne, facilite la vie des touristes (Clary, 1993 ; Duhamel et Violier, 2009).

$6 \mathrm{Au}$ contraire, la découverte suppose d'affronter des lieux historiquement constitués et marqués par l'altérité sociale, sauf lorsqu'elle se pratique sous la forme d'un succédané totalement artificiel comme dans quelques parcs à thème dans lesquels les entrepreneurs ont choisi de mettre le monde en scène (le parc à thème Windows of the World à Shenzhen en Chine par exemple qui offre un panorama complet du monde en quelques reproductions de grande dimension des principaux sites et édifices). La découverte suppose donc pour le touriste une soumission volontaire à l'altérité, car il mesure bien les risques encourus même si, chez l'individu, la dimension de jeu voire de pari n'est pas à exclure, puisque le frisson éprouvé contribue à la satisfaction retirée, pas plus qu'une certaine dose d'insouciance. Mais là encore des formes de médiation soutiennent les touristes dans les confrontations à l'altérité. Ainsi les tour-opérateurs ont pour fonction de permettre aux touristes de circuler à travers le monde. Les voyages organisés en sont les outils privilégiés. Cette technologie spatiale ne peut-être réduite à la caricature qui en est souvent faite et qui stigmatise la pérégrination de groupes dépossédés de leurs capacités d'acteurs. En fait, il est stratégique d'y avoir recours lorsqu'on ne dispose pas du capital, aussi bien spatial qu'économique, de s'en passer, car le voyage tout compris est proposé sur le marché à des prix plus bas. Il existe par ailleurs une grande variété de voyages plus ou moins organisés avec un gradient variable d'autonomisation des individus depuis le circuit tout compris et complètement anticipé jusqu'à des formules qui ne prévoient que le transport initial et l'hébergement, laissant au touriste la possibilité d'opter sur place pour des loisirs choisis au dernier moment.

7 Cette variété est l'un des signes que le tourisme évolue en relation avec le contexte social au sein duquel il se déploie. Il est en effet infléchi par l'individualisation. Les formes autonomes de la découverte tendent à se développer au détriment des formes encadrées du moins de manière relative. Il n'existe pas de mesure chiffrée de cette évolution, et les statistiques officielles ne permettent pas réellement de mesurer ces évolutions, et ce pour plusieurs raisons. Premièrement, le tourisme n'est pas réellement bien circonscrit par les statistiques officielles. En effet, les institutions qui les produisent se fondent, derrière l'appellation "tourisme", sur une définition globale des mobilités qui ne permet pas d'isoler les touristes tels que nous les avons définis (voir à ce propos Équipe Mit, 2002 ; Stock, 2003). En effet, l'Organisation mondiale du tourisme considère ainsi que sont 
touristes tous les individus qui passent une nuit en dehors de leur résidence principale quel que soit «le motif» du déplacement, ce qui inclut donc les pèlerinages, les voyages d'affaires... et même une catégorie " autre motif ». Or la prise en charge des individus par les organisations varie selon les modes de mobilité distingués en fonction de l'intention de l'individu mobile. En effet, dans les mobilités d'affaires, tout est soigneusement organisé par l'entreprise de laquelle relève l'individu. Ensuite, si nous disposons de statistiques sur le dénombrement des clients des tour-opérateurs en fonction de la destination (produites annuellement par le magazine spécialisé L'Écho touristique), elles ne sont pas exhaustives et nous n'avons pas accès à la ventilation précise par mode de pratique (voyage organisé tout compris, sur mesure organisé, vol sec...). Par ailleurs, même si nous disposions de la proportion de vols secs, achat par le touriste des billets d'avions sans autre prestation, ce qu'une partie des tour-opérateurs livre, rien ne garantit que le touriste n'a pas recours une fois sur place à une agence dite réceptive, avec laquelle il aura pris contact par internet et qui assurera son encadrement au moins partiel ${ }^{3}$.

Nous pouvons donc seulement relever des indices d'autonomisation croissante des touristes, comme la diversification des propositions émanant des tour-opérateurs, du moins de ceux qui ne fondent pas leur stratégie sur les prix, lesquels du coup privilégient le voyage tout compris qui permet les économies d'échelle. À l'inverse en effet, la stratégie contraire qui consiste à tirer partie de la valeur ajoutée élevée plus que du volume s'accompagne de propositions qui tendent à laisser aux touristes une marge de manœuvre significative dans l'élaboration du projet touristique. Le «package dynamique " permet ainsi au touriste d'élaborer pas à pas son propre circuit touristique en combinant sur internet des propositions de transports et d'établissements d'hébergement dûment répertoriées par le tour-opérateur et en maitrisant la durée des étapes. Mais cette possibilité n'existe que pour un nombre limité de destinations, les unes marquées par une faible altérité pour des touristes donnés, les autres par une forte maturité touristique caractérisée par une accumulation d'arrangements favorables à la territorialisation des touristes. Le tour-opérateur Kuoni positionné sur le haut de gamme ne proposait ainsi en 2014, au départ de la France, que quelques destinations : les ÉtatsUnis et le Canada dans la première catégorie, la Thaillande dans la seconde.

L'individuation soutient donc la tendance des touristes à s'affranchir des organisations pour se confronter à l'altérité. De ce fait, la question du capital spatial différencié qui permet à des individus de mettre en œuvre un projet de découverte touristique autonome est posée. Mais également, et le présent article s'intéresse à cet autre versant, nous pouvons formuler l'hypothèse que des arrangements spatiaux peuvent être mis en œuvre dans les espaces et lieux touristiques afin de construire une familiarité partielle, par distinction avec la familiarité très poussée observable dans les comptoirs. En effet, il ne s'agit pas de la production intégrale d'un lieu fonctionnel conforme aux normes des sociétés dont les touristes sont originaires, mais d'ajustements limités. Ces derniers créent suffisamment de familiarité pour que le touriste circule de manière autonome, mais ils n'abolissent en rien l'altérité ; du reste la pratique même de la découverte repose sur le principe d'un différentiel sans lequel le voyage serait dévalorisé. D’ailleurs cela ne serait pas possible, puisque le lieu reste approprié par ses habitants.

Pour mettre en œuvre une expérimentation qui permette de valider la proposition qu'une stratégie des acteurs territorialisés peut construire de la familiarité, nous proposons de préciser plus concrètement ce que nous entendons par altérité et de montrer comment les touristes y sont confrontés dans une pratique de découverte. Cette dernière consiste à 
parcourir des espaces structurés en réseaux joignant des lieux perçus comme exemplaires de la société à découvrir, et d'autres comme fonctionnels: ainsi des établissements d'hébergement ou des nœuds de transports tels qu'aéroports, gares, stations de métro ou de bus. Deux mobilités se succèdent donc : une première par laquelle le touriste accède à sa destination et une seconde qu'il met en œuvre dans l'espace qu'il a projeté de visiter.

L'expérimentation va porter sur cette mobilité seconde le long des itinéraires construits par les touristes qui revendiquent une valeur d'autonomie. Ceux-ci, pour réaliser leurs projets de découverte, peuvent avoir recours à différents modes de déplacement. Gwendal Simon (2010) a ainsi étudié à Paris les mouvements intra-urbains des touristes, mais s'il a analysé comment les individus procédaient avec la marche et avec le métro, il ne questionne pas les possibilités d'adaptation des itinéraires aux compétences de spatialité (Lussault, 2013) des touristes. Or pour les utiliser des informations sont nécessaires, et elles ne sont pas données mais construites par les acteurs gestionnaires de l'espace urbain pour la marche, donc les services de la voierie des villes, ou les entrepreneurs du métro. De même notre approche est complémentaire de celle mise en œuvre par Émilie Flon, car si nous pouvons mobiliser le concept d'adhérence, lequel «se définit par le degré et le mode d'inscription urbaine d'un type de mouvement ", c'est en le déformant. En effet, l'auteure le "définit [comme] un outil pour reconnecter la signification du transport et des activités : plus un mode de transport "adhère" à la ville, plus il est imbriqué aux activités et à l'environnement spécifiquement urbains, plus il favorise les premières et enrichit les échanges avec ce dernier " (Flon, 2012, p. 91), alors que nous le convoquons dans une approche qui vise à appréhender la maîtrise du déplacement par les touristes et non à analyser le réenchantement de la ville. Elle porte sur l'accès à ces moyens et sur la maîtrise des réseaux qui permet de repérer puis pratiquer un itinéraire. Nous allons donc analyser comment, dans différents lieux touristiques, des arrangements spatiaux permettent la circulation autonome des touristes en leur procurant des prises (Joseph, 1994 et 1998) ou affordances (Gibson, 1986), concept qui articule l'objectif, les moyens mis en œuvre, et le subjectif, l'individu qui s'en saisit. De manière concrète nous allons mener cette expérimentation à partir des réseaux de métro de différentes villes du monde.

Pour préparer et réaliser ces itinéraires, les touristes sont confrontés aux signes et aux dispositifs qui les jalonnent. Dans cette perspective les différences linguistiques exercent un rôle d'écran et opposent leurs rugosités à des pratiques autonomes. Différents degré d'altérité peuvent être repérés qui sont relatifs aux individus et sociétés. Aussi le tableau 1 est établi pour un individu occidental. Les signes utilisés peuvent être des symboles dont la compréhension est transculturelle, mais on n'échappe pas à l'écrit ou aux messages enregistrés qui sont véhiculés par des langues. Ces dernières sont plus ou moins diffusées parmi les touristes, notamment grâce aux apprentissages scolaires que ces derniers peuvent investir dans leurs pratiques. De ce point de vue, nous pouvons dégager un premier gradient : la capacité du touriste à circuler dans les villes du monde dépend de la fréquence de l'apprentissage de la langue utilisée dans l'espace visité (seconde colonne). Cette proposition se subdivise en deux: langue(s) dominante(s) d'usage dans le lieu d'une part, fréquence des traductions dans une ou d'autres langues. Ensuite, à défaut de la maîtrise par un apprentissage antérieur de longue durée, le touriste peut tenter d'apprendre rapidement, et dans l'objectif du déplacement, des rudiments - des méthodes variées existent -, voire se munir seulement d'un dictionnaire basique et tenter la lecture et la traduction des informations de base. Dans cette 
démarche courageuse, le touriste se heurte à une complexité variable des systèmes de signes. En effet, s'il est aisé pour un européen occidental de lire des inscriptions dans une langue latine autre que la sienne, cela se complique dès lors que l'alphabet est cyrillique ou que l'écriture est constituée d'idéogrammes. Apparaît donc un second gradient. Nous pouvons en déduire que la mobilité touristique se heurte à une rugosité qui combine ces différentes dimensions.

Tableau 1. Degré de familiarité des langues écrites selon l'apprentissage et le système d'écriture

\begin{tabular}{|l|l|l|l|}
\hline Langues & Fréquence de l'apprentissage & Système d'écriture & Rugosité \\
\hline Anglais & ++++ & Alphabet latin & --- \\
\hline Espagnol & +++ & Alphabet latin & -- \\
\hline Allemand & ++ & Alphabet latin & -- \\
\hline Portugais & + & Alphabet latin & -- \\
\hline Russe & - & Alphabet cyrillique & ++ \\
\hline Chinois & -- & Idéogrammes & +++ \\
\hline
\end{tabular}

\section{Expérimentation par analyse des technologies spatiales des métros de différentes villes du monde}

13 L'expérimentation est réalisée à propos du métro. Il semble plus aisé, à condition que l'opérateur du réseau en ait saisi l'enjeu, de circuler en métro, moyen par lequel le touriste peut espérer être autonome, qu'en taxi qui nécessite d'entrer en communication avec le chauffeur. Par ailleurs le métro est devenu une technologie spatiale intégrée par un grand nombre d'urbains dans leur quotidien et participe d'un capital spatial qui est régulièrement mis à l'épreuve dans la pratique touristique des grandes villes. Dans sa thèse (2010), Gwendal Simon souligne ainsi que le métro est, après la marche à pied, le moyen le plus utilisé par les touristes à Paris. La promenade est délaissée à son profit dès que la distance à franchir, pour accéder à l'objet dont la visite est projetée, est perçue comme élevée. Cette faculté d'anticipation relève de compétences spatiales diverses (Lussault, 2013), notamment la compétence métrique qui permet de mesurer les distances, la compétence de parcours qui permet de composer un itinéraire, et la maitrise spatiale qui rend capable de découper un séjour touristique en unités (jour ou demijournée).

14 L'expérimentation a pour but d'évaluer comment les acteurs locaux facilitent l'usage de leur métro par les touristes, afin de vérifier l'hypothèse qu'une familiarité construite permet une relativisation fonctionnelle de l'altérité et donc autorise la mobilité autonome. Au-delà, cela signifie que par le tourisme, en raison des enjeux économiques qui lui sont liés, se construit un horizon mondial de mobilité; et cela illustre le rôle mondialisant du tourisme qui n'est pas seulement mondialisé, c'est-à-dire diffusé dans le monde, mais aussi agent de la mondialisation. Pour mettre en œuvre l'expérimentation, nous avons renoncé à mobiliser les méthodes plus classiques des sciences sociales, à savoir l'enquête par questionnaire ou entretien, en raison des obstacles auxquelles elles se heurtent. En effet, les conditions de mise en œuvre d'une interrogation aléatoire sont 
quasi impossibles à atteindre dans un espace clos et étroit au sein duquel des individus se pressent et se déplacent rapidement. Ensuite, comme nous souhaitons saisir les situations problématiques au moment où elles se produisent, et non après qu'elles aient été ou non surmontées, ou à des moments où les personnes sont en mesure de réévaluer leur expérience vécue, le risque de ruse ne peut être écarté. Nous avons donc choisi de conduire une observation de type autoethnographique (Brougère, 2014) en utilisant non pas un journal, au sens strict, mais un reportage photographique réalisé pour apprécier l'expérience touristique dans les métros de différentes villes du monde.

Une grille d'observation a été préalablement élaborée afin d'objectiver l'observation. Elle a été bâtie selon les opérations réalisées pour circuler en métro. Nous en avons sélectionné trois qui seront étudiées dans différentes villes qui ont en commun d'être des grandes destinations touristiques (Duhamel et Knafou, 2007) : Berlin, Saint-Pétersbourg, Moscou, Shanghai et New-York. Ces trois approches restituent l'expérience des touristes qui veulent circuler par le métro : l'accès au métro, à savoir l'acquisition des billets et le franchissement des barrières antifraude, ensuite le repérage dans le réseau soutenu par des informations diffusées dans les stations et dans les rames, et enfin la circulation dans les stations (accès aux lignes et sorties vers la ville). Le tableau 2 résume les observations effectuées. Deux niveaux d'analyse ont été combinés. D'une part, l'évaluation de l'ensemble du réseau suppose de la part des touristes un apprentissage in situ qui, combiné avec le réinvestissement de l'expérience acquise, d'autant plus importante que l'individu est originaire d'une métropole équipée, débouche une fois tentée une première expérience sur un savoir-faire localisé. D'autre part, les réseaux ne sont pas totalement homogènes et le touriste devra ici et là s'adapter à des particularismes. Les villes ont été sélectionnées en raison de l'intensité de leur fréquentation, soit à partir de déplacements touristiques que nous avons effectués, soit à l'occasion de mobilités professionnelles mais pendant lesquelles des moments touristiques nous ont permis de nous trouver en situation d'autonomie. Si nous affirmons que les mobilités d'affaires ne sont pas des déplacements touristiques au sens où le projet comme le programme échappent à l'individu mobile, il n'en demeure pas moins que les ruses des individus ou les occasions ${ }^{4}$ saisies par les donneurs d'ordre peuvent générer des moments, qualifiés de touristiques car inscrits dans le hors-quotidien, que l'individu peut saisir pour inscrire son projet personnel dans une organisation qui lui échappe au moins partiellement.

Tableau 2. Présentation synthétique des observations

\begin{tabular}{|c|c|c|c|c|c|c|}
\hline \multirow[b]{2}{*}{ Thèmes } & \multirow{2}{*}{$\begin{array}{l}\text { Sous- } \\
\text { thèmes }\end{array}$} & \multicolumn{5}{|l|}{ Villes } \\
\hline & & New-York & Berlin & $\begin{array}{l}\text { Saint- } \\
\text { Pétersbourg }\end{array}$ & Moscou & Shanghai \\
\hline \multirow{3}{*}{ Langages } & $\begin{array}{l}\text { Système } \\
\text { d'écriture }\end{array}$ & $\begin{array}{l}\text { Alphabet } \\
\text { latin }\end{array}$ & Alphabet latin & $\begin{array}{l}\text { Alphabet } \\
\text { cyrillique }\end{array}$ & $\begin{array}{l}\text { Alphabet } \\
\text { cyrillique }\end{array}$ & Idéogrammes \\
\hline & Langue & Anglais & Allemand & Russe & Russe & Chinois \\
\hline & Traduction & $\begin{array}{l}\text { Traduction } \\
\text { en espagnol } \\
\text { très } \\
\text { fréquente }\end{array}$ & $\begin{array}{l}\text { Traduction en } \\
\text { anglais très } \\
\text { fréquente }\end{array}$ & $\begin{array}{l}\text { Traduction } \\
\text { en anglais } \\
\text { fréquente }\end{array}$ & $\begin{array}{l}\text { Pas de } \\
\text { traduction }\end{array}$ & $\begin{array}{l}\text { Traduction } \\
\text { en anglais } \\
\text { fréquente }\end{array}$ \\
\hline
\end{tabular}




\begin{tabular}{|c|c|c|c|c|c|c|}
\hline \multirow{2}{*}{$\begin{array}{l}\text { Acheter } \\
\text { un billet } \\
\text { Connaître } \\
\text { les } \\
\text { formules } \\
\text { tarifaires }\end{array}$} & $\begin{array}{l}\text { Caisses } \\
\text { automatiques }\end{array}$ & Nombreuses & $\begin{array}{l}\text { Nombreuses } \\
\text { Multilinguisme }\end{array}$ & Non & Non & $\begin{array}{l}\text { Nombreuses } \\
\text { caisses } \\
\text { automatiques } \\
\text { Problème } \\
\text { d'achat en } \\
\text { pièces ou } \\
\text { billets de } \\
50 \text { yuan } \\
\text { maximum } \\
\text { Problème de } \\
\text { langue aux } \\
\text { caisses }\end{array}$ \\
\hline & $\begin{array}{l}\text { Caisses avec } \\
\text { personnel }\end{array}$ & Oui & Oui & $\begin{array}{l}\text { Des } \\
\text { formules à } \\
\text { tarif réduit } \\
\text { existent } \\
\text { mais } \\
\text { aléatoires } \\
(22 \\
\text { roubles / } \\
\text { jeton / } 20 \\
\text { par 10) }\end{array}$ & $\begin{array}{l}\text { Personnel ne } \\
\text { parle pas } \\
\text { anglais }\end{array}$ & $\begin{array}{l} \\
\text { Caisses peu } \\
\text { nombreuses } \\
\text { Pas de } \\
\text { bilinguisme }\end{array}$ \\
\hline
\end{tabular}




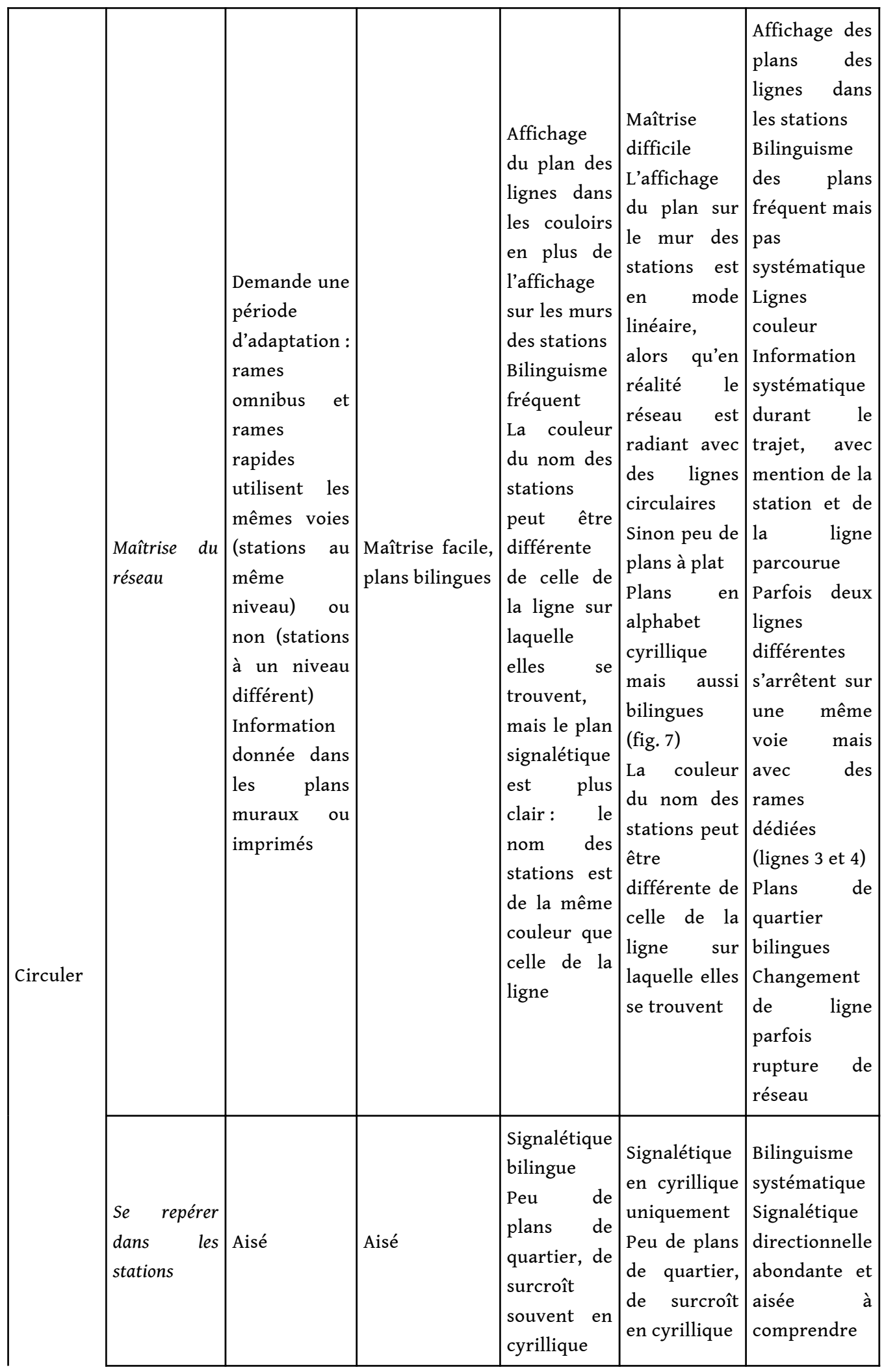




\begin{tabular}{|c|c|c|c|c|c|}
\hline $\begin{array}{lr}\text { Se repérer } \\
\text { dans les } \\
\text { rames }\end{array}$ & $\begin{array}{l}\text { Facile : } \\
\text { information } \\
\text { abondante } \\
\text { diffusée par } \\
\text { oral ou écrit }\end{array}$ & $\begin{array}{l}\text { Facile : } \\
\text { affichage } \\
\text { bilingue }\end{array}$ & $\begin{array}{l}\text { Information } \\
\text { dans les } \\
\text { rames } \\
\text { bilingues } \\
\text { sur plans } \\
\text { linéaires } \\
\text { Annonves } \\
\text { orales faites } \\
\text { en russe }\end{array}$ & $\begin{array}{l}\text { Information } \\
\text { dans les } \\
\text { rames } \\
\text { variable } \\
\text { Certaines } \\
\text { rames ont un } \\
\text { affichage } \\
\text { électronique } \\
\text { à l'intérieur, } \\
\text { sinon } \\
\text { affichage } \\
\text { non } \\
\text { électronique, } \\
\text { ou encore } \\
\text { aucun } \\
\text { affichage } \\
\text { Annonces } \\
\text { orales faites } \\
\text { en russe }\end{array}$ & $\begin{array}{l}\text { Information } \\
\text { présente dans } \\
\text { les rames } \\
\text { (plan de la } \\
\text { ligne) } \\
\text { Indication de } \\
\text { la position de } \\
\text { la rame par } \\
\text { diodes } \\
\text { lumineuses } \\
\text { dans les } \\
\text { installations } \\
\text { les } \\
\text { récentes plus } \\
\end{array}$ \\
\hline
\end{tabular}

Une technologie spatiale très efficace mais qui, à notre connaissance, n'est en usage qu'à Beijing, aplanit les difficultés du taxi (fig. 1). Il s'agit d'une carte de format carte de visite qui est mise à disposition dans les hôtels. L'adresse de l'établissement figure dessus, ce qui assure au touriste de revenir à son point de départ. Ce dernier coche sur la carte, parmi les items préétablis, sa destination et tend la carte au chauffeur. Une version plus fruste de cette technologie est également diffusé dans d'autres villes. La carte n'indique que le nom et l'adresse de l'hôtel, avec un plan au verso, et la mention « Please take me to... [suit le nom de l'hôtel] ». Le touriste peut au moins rentrer à son hôtel! Nous aurions pu également réaliser l'expérimentation à propos du réseau de bus urbain. Les touristes peuvent aussi avoir recours aux circuits de bus touristiques qui desservent un circuit de points d'arrêt définis par avance et desservis en boucles. Dans ce cas, la concentration des touristes induite par la fonctionnalité permet la mise à disposition d'un personnel d'accueil qui assure la médiation. De tels circuits permettent d'accéder aux lieux les plus fréquentés. Le touriste aura donc recours aux moyens publics non spécialisés dès qu'il souhaitera visiter un établissement plus confidentiel ou rejoindre son hébergement pour peu que celui-ci ne soit pas situé dans le Central Tourist District, espace de touristicité maximale (Duhamel et Knafou, 2007), qui est généralement irrigué par les transports urbains touristiques spécialisés. 


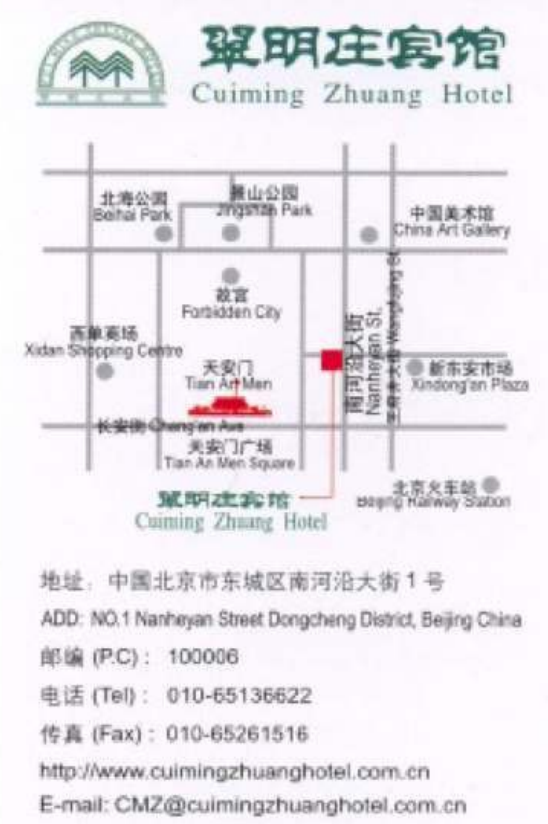

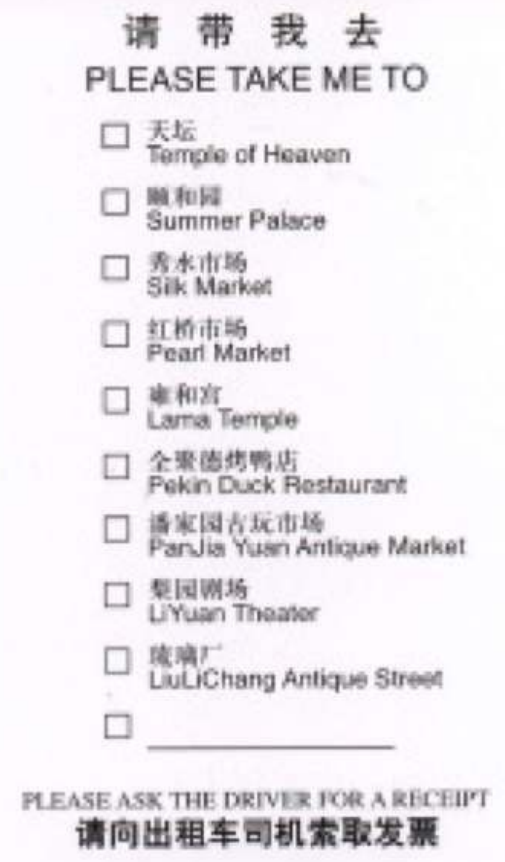

请 带 我 去 PLEASE TAKE ME TO

天坛

of Heaven

Gummer Palace

Marken

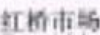

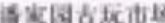

Pardia Yusi Antigue Market

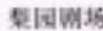

LYush Thearer

otray

LuLichang Antique Streed

请向出租车司㧈素取发票

Fiche cartonnée (format carte de visite) distribuée dans les hôtels que les touristes cochent et remettent au chauffeur de taxi à Beijing. Cette technologie permet une découverte autonome de la ville, c'est-à-dire sans recours aux agences réceptives ou aux voyagistes.

\section{Un monde habitable}

L'analyse synthétique met en exergue la combinaison entre deux ordres de facteurs: d'une part la diversité des structures des réseaux de métro, d'autre part la familiarité construite par les acteurs du lieu. En effet le savoir-faire des individus-touristes a été constitué à partir de l'expérience vécue au quotidien d'un réseau, appelée compétence d'usager (Joseph, 1998). Leur apprentissage est donc structuré en fonction d'un certain type de réseau, par exemple le métro de Paris pour les personnes qui résident en France. Il y a donc un seuil d'altérité à franchir qui relève de l'adaptation à un réseau différent. Dans le second facteur, la construction de la familiarité joue à plein. Dans cette dimension, les individus touristes se heurtent à l'altérité du lieu.

\section{Le transfert de la compétence d'usager}

Le touriste le plus expérimenté, dans le cas de cette étude l'usager rompu à la pratique du métro d'une métropole, devra s'adapter aux particularités du réseau de la ville visitée. À New York l'usage du métro est aisé puisque toutes les informations sont diffusées en anglais aussi bien qu'en espagnol, cependant la morphologie urbaine induit celle du réseau. Dans la partie intensément fréquentée par les touristes, des choix techniques ont été opérés qui constituent autant de particularités. Ainsi les lignes convergent-elles vers Manhattan, puis vers Downtown, et, dans ce centre de la métropole plusieurs d'entre elles, distinguées, en plus des couleurs, par des chiffres ou des lettres, utilisent les mêmes 
voies (notamment les lignes 4, 5 et 6 à l'est, ou les lignes 1, 2, 3 d'une part, et A, B, C, D et E d'autre part, à l'ouest). Elles sont parcourues chacune par des rames de type différent. Les unes, dans leur traversée de Manhattan, sont omnibus et s'arrêtent à toutes les stations (lignes 4 et 5) tandis que d'autres, express, ne s'arrêtent qu'à certaines stations (ligne 6). L'information est communiquée sur les plans abondamment diffusés. L'accès aux rames peut se situer selon les stations au même niveau ou non. Cette dernière information est signalée de manière efficace dans les stations si bien que cette particularité peut être assez aisément maîtrisée (fig. 2). À Washington, c'est la tarification qui pose problème. D'une part elle varie selon les stations de destination, sans que la notion de zone apparaisse, et d'autre part selon les horaires. Le coût est plus élevé aux heures de pointe. La lecture du tableau à double entrée qui couvre la moitié de la surface des caisses automatiques est nécessaire au bon usage.

Figure 2. Signalétique dans la station de la $59^{\mathrm{e}}$ rue à New York

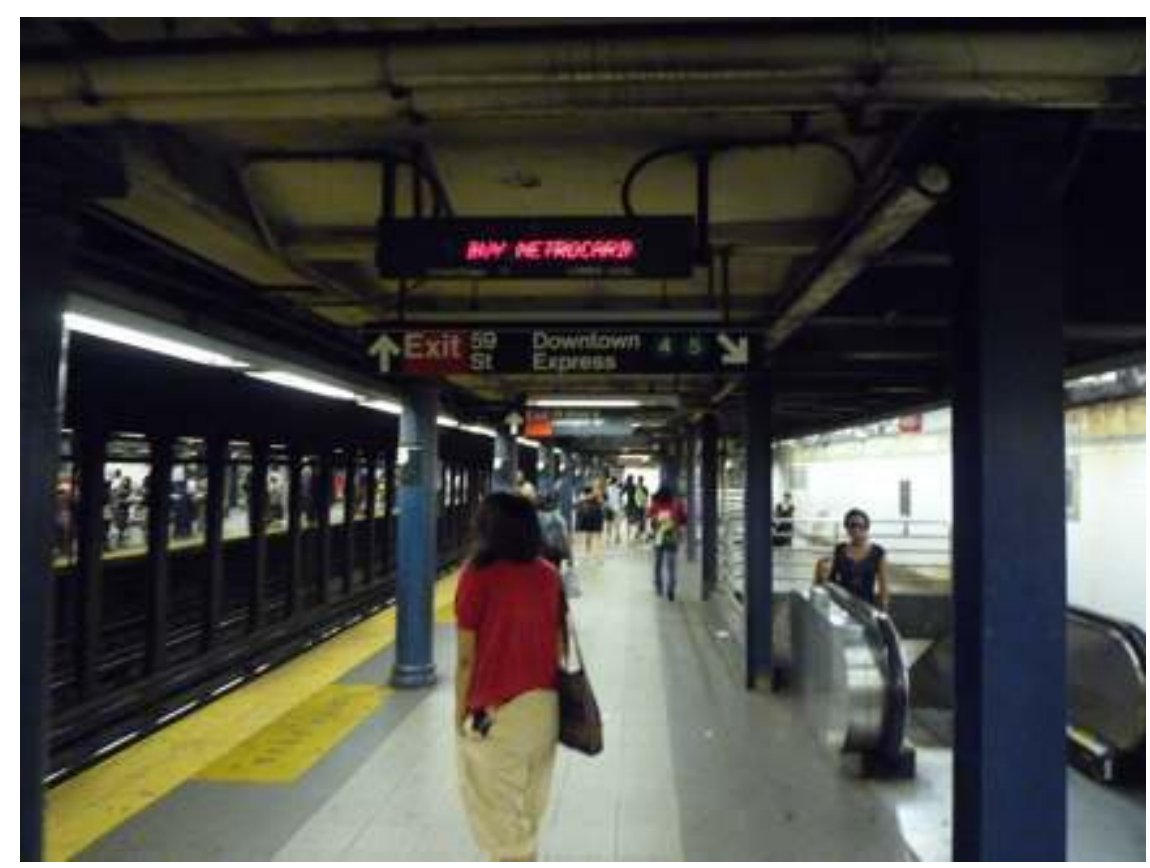

Il est clairement mentionné que le quai au premier plan permet d'accéder aux rames de la ligne 6 , express vers downtown, alors que l'accès aux rames des lignes 4 et 5 s'effectue au niveau inférieur accessible par l'escalator situé à l'arrière-plan à droite. La sortie est également clairement mentionnée.

Photo P. Violier, 27 juillet 2010.

19 Les réseaux de Moscou et Saint-Pétersbourg recèlent aussi certaines particularités qui n'en facilitent pas l'usage au Parisien le plus expérimenté en savoir-penser et savoir-faire métropolitains. En effet, les noms des stations varient selon la ligne, comme le montre le tableau 3, alors que dans le métro parisien la station est nommée de manière identique quelle que soit la ligne empruntée. Cela, combiné avec l'usage de l'alphabet cyrillique, complique sérieusement les changements pour le néophyte. Cependant, une autre particularité du métro moscovite se révèle à l'usage profitable au touriste. En effet, dans les stations moscovites les quais sont situés au centre et les rames en périphérie. En cas d'erreur au sujet de la direction le touriste pourra vite changer de rame, alors que dans la 
plupart des stations parisiennes il faut retourner en sens inverse, passer au-dessus du tunnel pour emprunter la rame dans l'autre sens.

Tableau 3 : Variation du nom des stations selon la ligne dans le réseau du métro de Moscou à une connexion qui permet l'accès au Kremlin

\begin{tabular}{|c|c|c|c|c|c|}
\hline \multicolumn{2}{|l|}{ Nom de la ligne } & \multicolumn{2}{|c|}{ Nom de la station } & \multirow{2}{*}{\begin{tabular}{|l} 
Couleur \\
de la \\
ligne
\end{tabular}} & \multirow{2}{*}{$\begin{array}{l}\mathrm{N}^{\mathrm{o}} \mathrm{de} \\
\mathrm{la} \\
\text { ligne }\end{array}$} \\
\hline En alphabet latin & En alphabet cyrillique & $\begin{array}{ll}\text { En } & \text { alphabet } \\
\text { latin } & \end{array}$ & $\begin{array}{l}\text { En alphabet } \\
\text { cyrillique }\end{array}$ & & \\
\hline Filiovskaïa & ФИЛИОВСКАїА & $\begin{array}{l}\text { Alexandrovski } \\
\text { Sad }\end{array}$ & $\begin{array}{l}\text { АЛЕХАНДРОВСКИ } \\
\text { САД }\end{array}$ & $\begin{array}{l}\text { bleu } \\
\text { clair }\end{array}$ & 4 \\
\hline $\begin{array}{l}\text { Serpoukhovsko- } \\
\text { Timiriazievskaïa }\end{array}$ & $\begin{array}{l}\text { СЕРПОУКХОВСКО- } \\
\text { ТИМИРИАЗИЕВСКАЇА }\end{array}$ & Borovitskaïa & БОРОВИТСКӒ̈А & gris & 9 \\
\hline Sokolnitcheskaïa & СОКОЛНИТЧЕСКАЇА & $\begin{array}{l}\text { Biblioteka } \\
\text { Imeni Lenina }\end{array}$ & $\begin{array}{l}\text { БИБЛИОТЕКА } \\
\text { ИМЕНИ ЛЕНИНА }\end{array}$ & rouge & 1 \\
\hline $\begin{array}{l}\text { Arbatsko- } \\
\text { Pokrovskaïa }\end{array}$ & $\begin{array}{l}\text { АРБАТСКО- } \\
\text { ПОКРОВСКАїА }\end{array}$ & Arbatskaïa & АРБАТСКӒ̈А & $\begin{array}{l}\text { bleu } \\
\text { foncé }\end{array}$ & 3 \\
\hline
\end{tabular}

Figure 3 : Signalétique dans le métro de Moscou

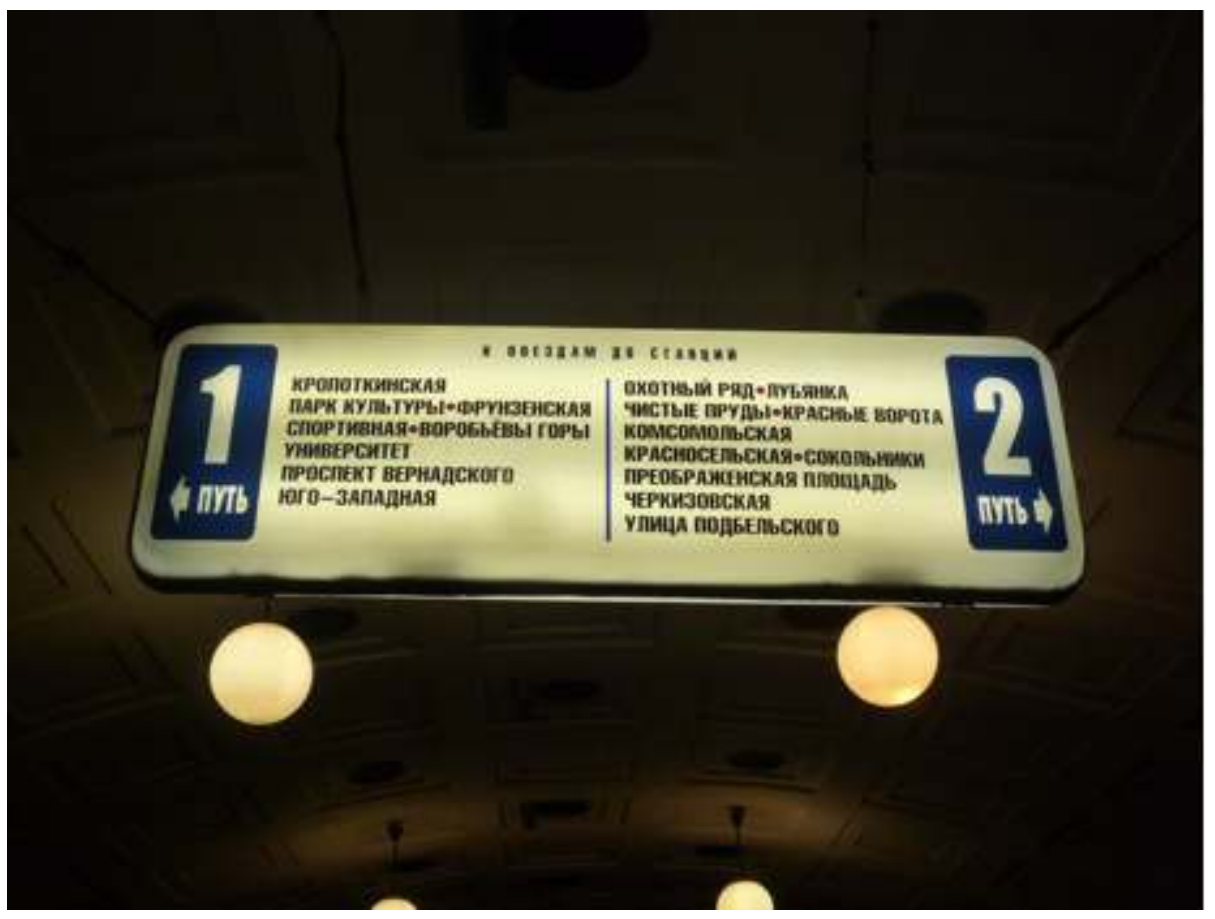

Un panneau lumineux indique les stations desservies par les rames de la ligne rouge (voir tableau 3). Cela révèle également le dispositif de quai central séparant les deux voies ou itinéraires (ПУТЬ) 1 et 2 , avec les stations desservies dans chaque sens.

Photo P. Violier, 14 avril 2010

20 Le réseau de Shanghai s'avère facile à utiliser. Sa structure est proche de celle du métro de Paris. Toutefois quelques embûches guettent le passager distrait. Les lignes 3 et 4 partagent pendant une partie importante de leur parcours la même voie. Cependant, les 
rames portent de manière distincte les numéros des lignes et les couleurs qui les distinguent (fig.4). Autre cas, la ligne 2 est censée relier directement l'aéroport international de Pudong et l'aéroport domestique Hongqiao en passant par la place du Peuple, où sont situés plusieurs destinations hautement touristiques comme le musée de Shanghai, le musée de la ville ou l'opéra. Or cette ligne est interrompue et le changement de rame est nécessaire à la station de Guanglan.

Figure 4 : Rame du métro de Shanghai

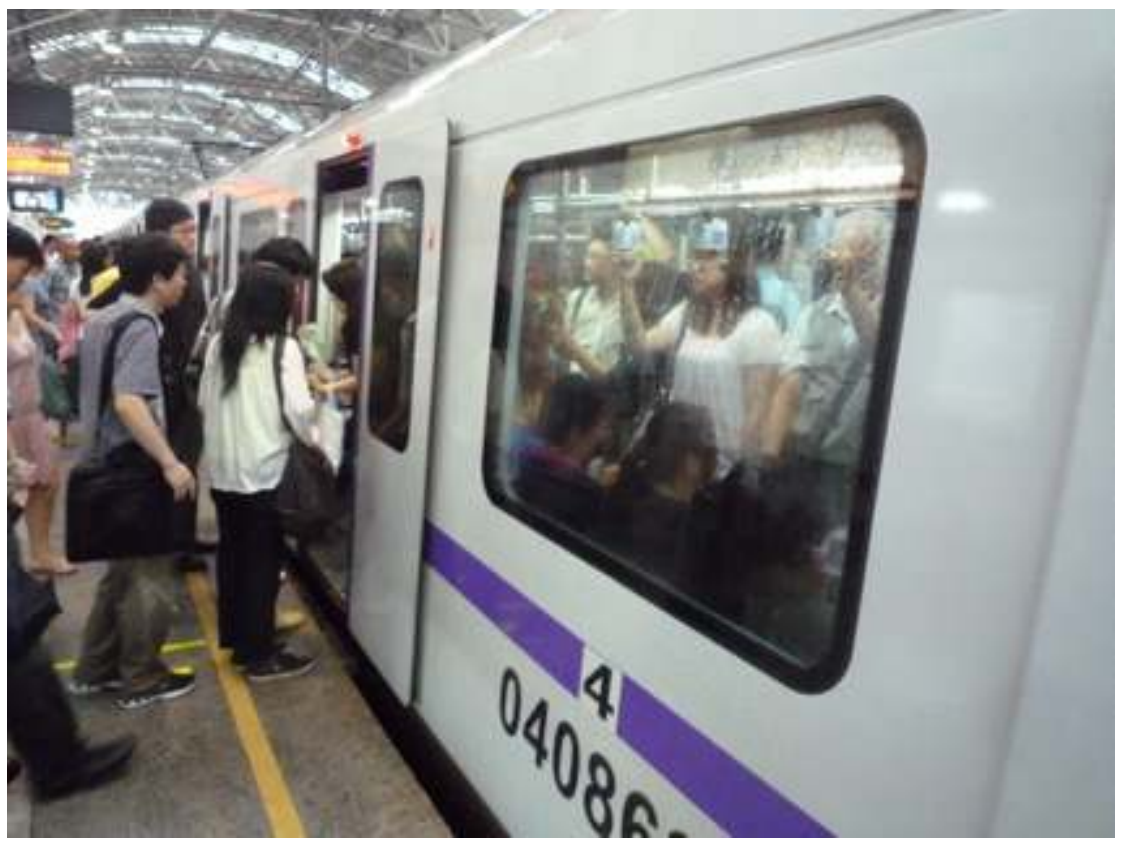

Le numéro de la ligne est clairement indiqué sur le flanc du wagon (4, au premier plan), ce qui permet d'informer le voyageur sur cette voie empruntée alternativement par des trains des lignes 3 et 4.

Photo P. Violier, 29 juin 2010.

Les transferts de savoir-faire d'un réseau à l'autre relèvent de la compétence des individus et nous renvoient donc à une des approches de la confrontation à la diversité du monde centrée sur la capacité individuelle comme évoqué en introduction. Nous allons maintenant aborder la manière dont la familiarité construite joue sur l'accessibilité.

\section{La construction de la familiarité}

L'expérimentation montre ensuite que le gradient de familiarité construite ne correspond pas à celui de l'altérité objective. En effet, entre les mondes, l'altérité maximale oppose bien aux Occidentaux les civilisations sinisées représentées ici par Shanghai. Car la Chine oppose une culture spécifique, qui a été seulement atténuée à la marge par des contacts limités avec l'Occident, à la différence du monde indien par exemple. La Russie figurerait en situation médiane puisque des particularismes affirmés, comme la religion orthodoxe, contrarient l'intégration du lieu dans le monde européen occidental. Les analyses de la diversité du monde continuent à distinguer un ensemble russe après la dislocation de l'espace soviétique (Huntington, 1996). L'usage de l'alphabet cyrillique constitue, nous l'avons déjà vu (tableau 3 ; fig. 3), une spécificité très active dans le champ de notre investigation. Une représentation simple situerait donc sur un axe d'altérité croissante les destinations européennes occidentales à une extrémité et les villes chinoises à l'autre, 
les russes se tenant à mi-chemin. Or, le gradient d'altérité ressentie, cette dernière résultant de la différence entre l'altérité objective et la familiarité construite mesurée dans notre expérimentation, est plus élevé pour des touristes occidentaux dans le cas de Moscou, et dans un degré un peu moindre de Saint-Pétersbourg, que dans celui de Shanghai.

Le métro de Saint-Pétersbourg est plus accessible que celui de Moscou car des plans et des indications bilingues russe-anglais sont répandus. Mais dans les deux villes russes, les caisses automatiques ne sont pas en usage (fig. 5). L'accès au mode de transport exige donc de communiquer en russe avec des personnels qui ne maitrisent aucune langue étrangère. L'affichage des tarifs également en russe n'est d'aucun secours. Le langage des signes permet seul de se munir des précieux laissez-passer.

Figure 5. Pas de caisses automatiques dans le métro de Moscou...

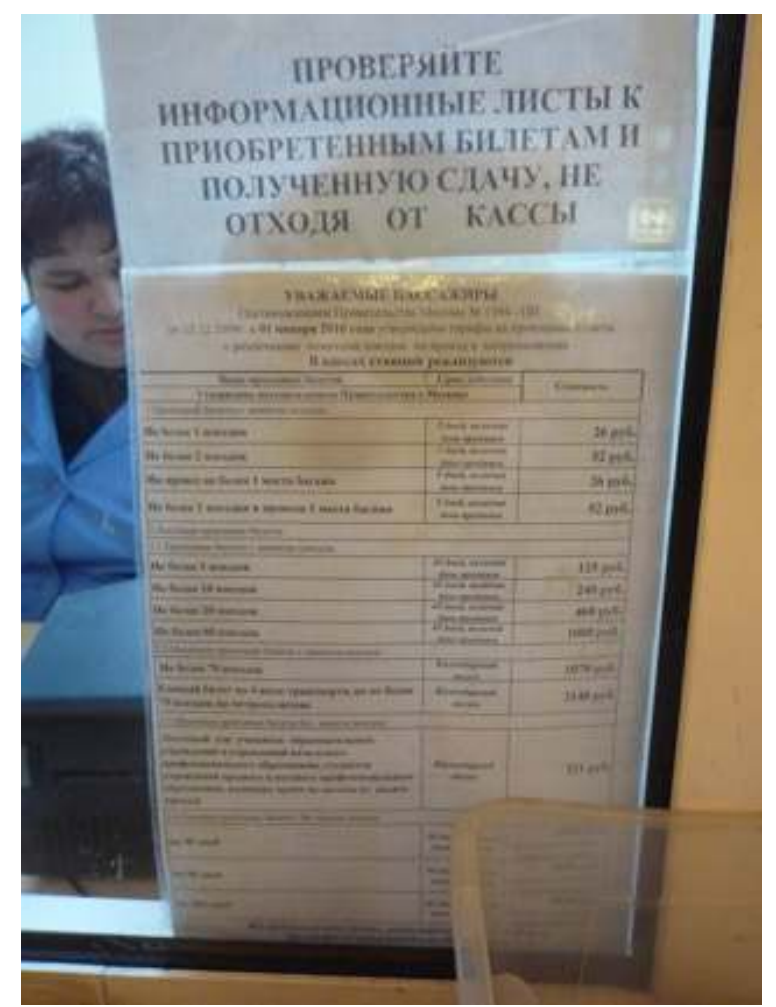

Pas de caisses automatiques dans le métro de Moscou, et un affichage aux caisses uniquement en russe, avec un personnel ne maîtrisant pas l'anglais.

Photo P. Violier, 14 avril 2010

Ce qui étonne surtout, c'est l'absence totale de traductions dans le métro moscovite, sa relative rareté dans celui de Saint-Pétersbourg, alors même que ces villes sont fréquentées par les touristes et que des événements internationaux y sont régulièrement organisés. Les plans ne sont pas bilingues à Moscou mais le sont à Saint-Pétersbourg, en particulier dans le centre historique, ce qui importe aux touristes. Cependant l'accès aux sites périphériques n'est pas aisé. Comme dans la plupart des destinations urbaines d'importance, l'espace touristique est multiple (Duhamel et Knafou, 2007) et inclut des établissements éloignés du centre. Plusieurs lieux historiques (Peterhof, Cronstadt) sont ainsi localisés dans la banlieue de Saint-Pétersbourg et accessibles par transport routier à 
partir des stations périphériques des lignes de métro. L'aventure est compliquée par l'absence de traduction des informations permettant le transfert entre le métro et les bus périurbains. De même, la circulation dans les métros se heurte à l'utilisation systématique à Moscou, plus mesurée à Saint-Pétersbourg, du seul alphabet cyrillique (fig. 6 et 7). Les changements de ligne et le choix des voies dans les stations, comme le repérage de la progression des rames sont dès lors délicats à opérer. D'autant que, comme nous l'avons vu précédemment, cela se conjugue avec une particularité des réseaux qui consiste à varier la nomination des stations selon les lignes (tableau 3 supra), ce qui complique singulièrement la tâche, notamment dans les immenses stations du centre-ville.

Figure 6 : Signalétique directionnelle à Moscou

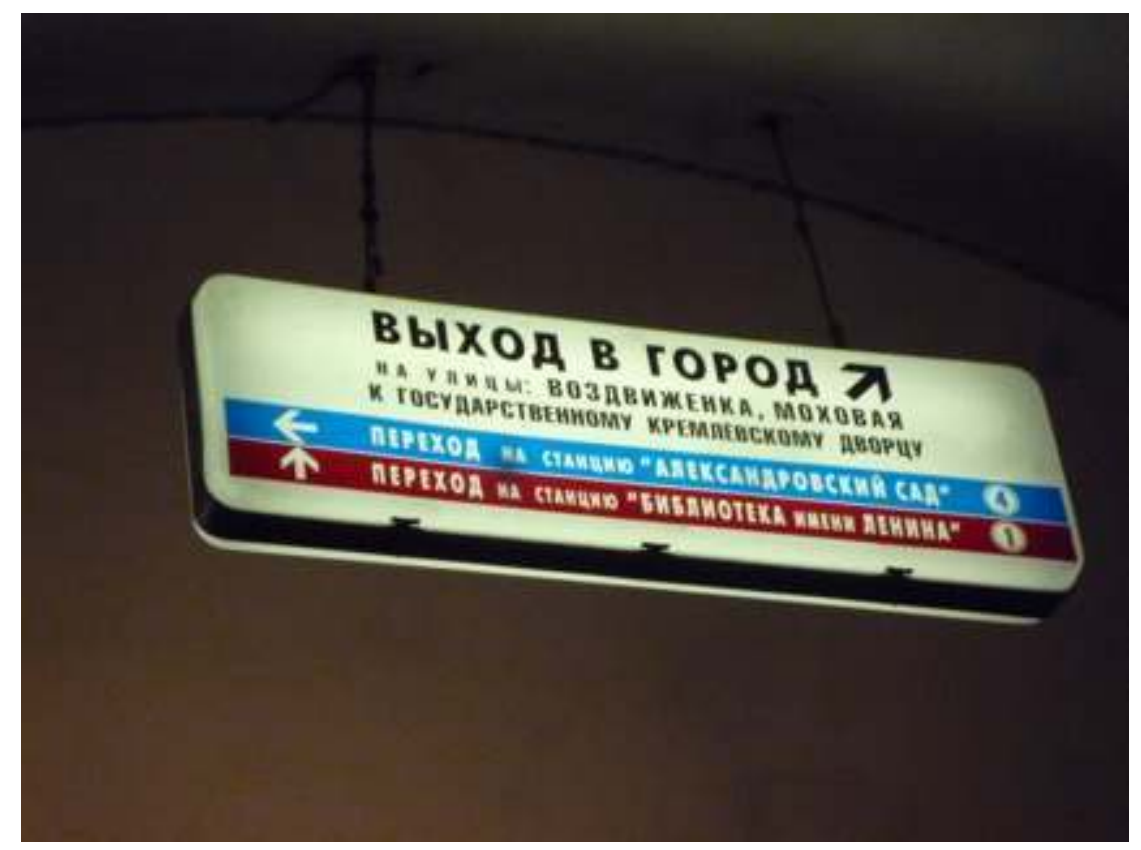

Signalétique directionnelle : en haut en noir, indication de la sortie ; en bas en bleu et rouge, les directions vers les deux lignes, la $n^{\circ} 4$, bleu clair, vers la gauche, la $n^{\circ} 1$, rouge, tout droit.

Photo P. Violier, 16 avril 2010. 


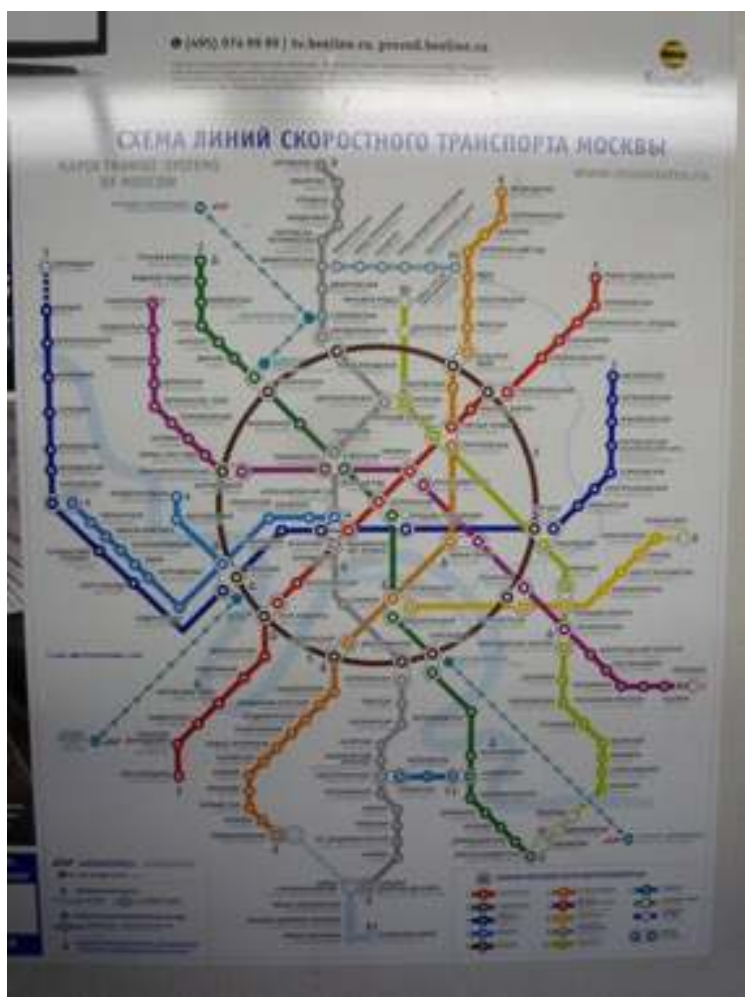

La transcription en alphabet latin sur le plan est de peu d'utilité tant que la signalétique du réseau (nom des stations, des lignes...) demeure en cyrillique.

Photo P. Violier, 16 avril 2010.

Or Moscou rivalise avec Saint-Pétersbourg comme première ville visitée de Russie, devenue une des premières destinations dans le monde. Le pays figure en effet au neuvième rang en 2007, selon l'Organisation mondiale du tourisme, avec près de 23 millions de voyageurs ${ }^{5}$ non résidents reçus (à comparer avec les 4 à 8 millions des années 1980). Qui plus est, le métro moscovite constitue un établissement touristique renommé. Construit dans les années 1930 , Staline a voulu en faire un symbole du régime et de nombreuses stations sont si richement décorées que les touristes s'y pressent et qu'aucun guide imprimé n'omet de le citer (fig. 8). Ces paradoxes s'expliquent par la conception que les dirigeants ont du tourisme, à savoir un tourisme strictement encadré. Par exemple, s'il est possible de louer un appartement à Moscou pour une durée limitée (à partir d'une nuit), les tracasseries administratives sont décourageantes. Le visa est en effet complété sur place de la " registration », enregistrement auprès de la milice, dont se chargent les gestionnaires des hébergements commerciaux. 
Figure 8 : Le métro de Moscou comme objet touristique

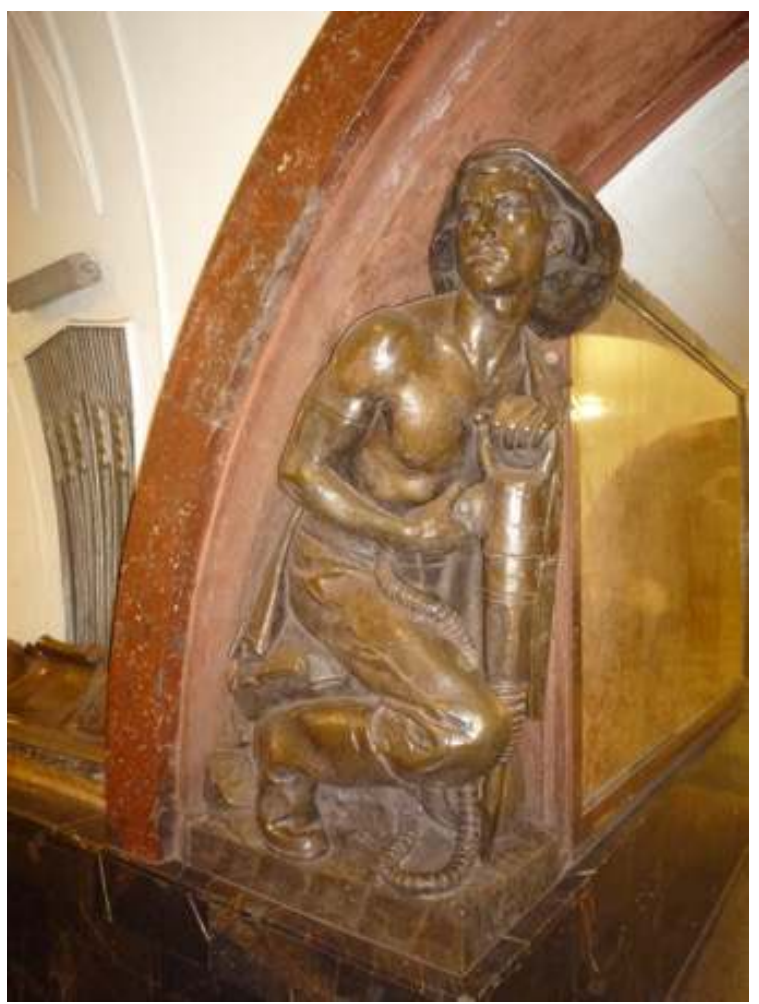

Le métro de Moscou constitue un objet touristique, ici station Park Koultouri ; pourtant l'accessibilité n'est pas facilitée pour les touristes.

Photo P. Violier, 11 avril 2010.

Inversement, l'usage du métro de Shanghai est d'une grande simplicité pour tout individu qui a acquis l'apprentissage de ce mode de transport. En effet, nous avons pu vérifier qu'un Chinois résident d'une ville dépourvue de métro s'y sentait moins à l'aise qu'un touriste habitué à ce mode de transport, et préférait recourir au service des taxis malgré les inconvénients du trafic surchargé. Les très nombreuses caisses automatiques facilitent l'accès au métro de cette ville (fig. 9). Elles proposent des informations en anglais. Une fois repéré sur le plan qui apparaît à l'écran la ligne et la station désirées, l'appareil calcule le coût en fonction du nombre de billets demandé. Il suffit alors d'introduire la monnaie pour récupérer les tickets. Le seul problème vient de ce que les caisses où l'on peut régler en billets sont plus rares que celles où l'on peut régler en pièces, et que les coupures de 100 yuan et plus ne sont pas acceptées. Dans ce cas il faut se diriger vers de rares caisses avec personnel, lequel ne s'exprime pas en anglais, mais comprend vite le problème face au billet tendu ! 

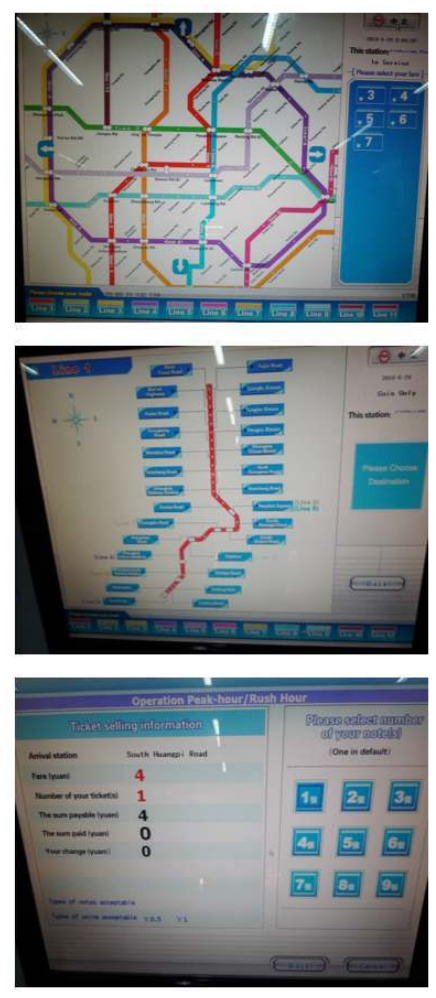

Les caisses automatiques facilitent l'usage du métro de Shanghai. Après avoir sélectionné sur l'écran tactile la version en anglais, le plan permet de choisir la ligne - sélection en bas du plan (a) -, puis la station de destination (b). L'appareil calcule alors le coût du voyage en tenant compte éventuellement du nombre de billets nécessaires (c).

Photos P. Violier, 29 juin 2010.

Ensuite la circulation dans les stations est extrêmement bien indiquée. La comparaison entre les écritures en anglais et en chinois souligne l'avantage procuré aux touristes autonomes par la traduction. Des panneaux très visibles et bilingues accrochés au plafond donnent les principales informations directionnelles qui sont parfois complétées par des indications fléchées au sol (fig. 10). Des plans de quartier indiquent aussi les sorties, toutes numérotées. Enfin, l'information sur les lignes, dans la station, sur les quais ou dans les rames est également bilingue et claire. Dans les stations les plus récentes, des jeux de couleurs indiquent la position de la station le long de la ligne, ce qui signale aussi la direction de la rame. Dans les rames de métro, les lignes sont également indiquées. Un affichage électronique bilingue et un enregistrement sonore annoncent les prochaines stations (fig. 11). Dans une rame de la ligne $\mathrm{n}^{\circ} 2$, qui joint les deux aéroports (Pudong Airport, aéroport international, et dédié aux lignes intérieures) et assure également l'accès aux espaces les plus touristiques de la ville (place du Peuple, rues de Nanjing, Bund, Pudong...), un affichage par diodes lumineuses indique la progression de la rame le long de la ligne, comme dans les rames les plus récentes du métro de Paris. Il en est de même d'ailleurs dans d'autres villes de Chine. Dans les métros de Beijing comme de Canton, l'usage du bilinguisme dans ses formes d'expression écrite ou orale est très largement répandu. 
Figure 10. Signalétique au croisement des lignes 4 et 7 à la station de Dongshan Road South

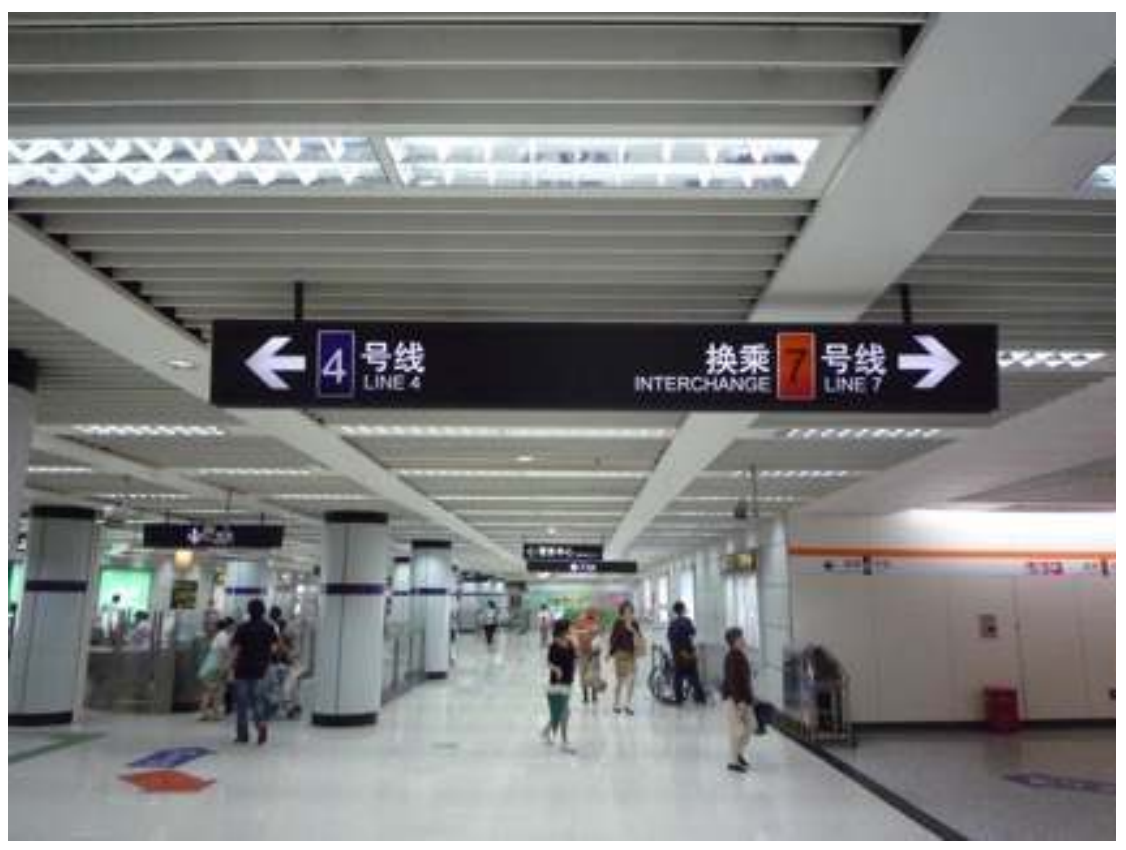

Outre la qualité de la signalétique, cette photo souligne également les atouts d'une construction récente, quand on compare l'espace vaste et clair de la station à l'exiguïté et à l'atmosphère glauque de nombreuses stations du métro de New York - fig. 2.

Photo, P. Violier, 29 juin 2010. 
Figure 11 : Information dans les rames à Shanghai

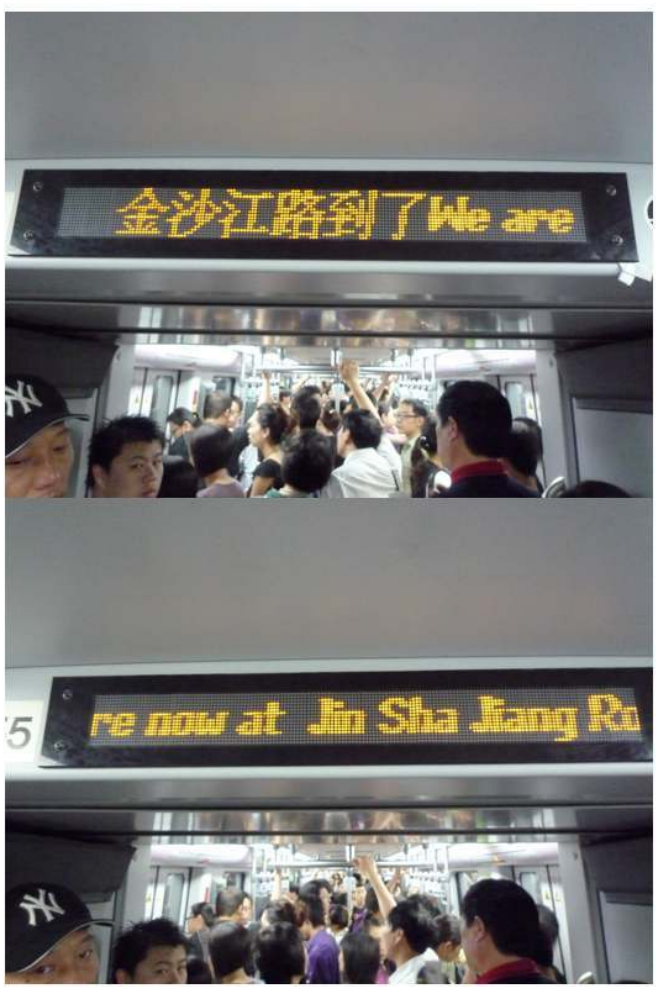

Information dans les rames par affichage lumineux bilingue (ici « [You a]re now at Jin Sha Jiang Road») et annonce sonore.

Photo P. Violier, 29 juin 2010.

Toutefois tout n'est pas parfait. S'il est aisé de circuler dans le métro de Shanghai, il est plus compliqué de sortir des stations pour rejoindre les objets touristiques convoités. En effet, dans les vastes stations du centre-ville, l'espace touristique majeur est circonscrit entre le Bund, la place du Peuple et la vieille ville; aussi n'est-il pas rare que le touriste soit amené à hésiter entre plusieurs sorties - une dizaine place du Peuple -, et ne trouve pour se repérer que des plans... en idéogrammes.

\section{Conclusion}

L'hypothèse d'une familiarité construite est donc validée par cette expérimentation puisqu'il est plus facile d'utiliser le métro à Shanghai qu'à Moscou, alors que l'altérité objective est plus élevée en Chine. Ainsi les acteurs territorialisés des destinations touristiques interviennent dans l'autonomisation par une réduction du différentiel. Celleci porte non seulement sur les transports comme nous l'avons vu, mais également sur la signalétique urbaine, qui permet d'accéder aux objets touristiques convoités, et sur l'interprétation qui donne par des moyens divers du sens à ces mêmes objets. Ces actions sont soutenues notamment par l'apport économique escompté par le développement touristique, si bien que l'affirmation que le tourisme contribue à faciliter la circulation des individus peut-être posée. Nous soulignons là une des dimensions mondialisantes du tourisme. D'autres intentions peuvent éventuellement contribuer aussi à construire cette familiarité, comme celles soutenues par des valeurs d'accueil, de rencontre, 
d'enrichissement mutuel... voire par une prise de conscience du processus de construction du monde.

Au-delà, cette recherche s'inscrit dans l'analyse du capital spatial et des dispositions ou des compétences dont les individus disposent pour régler les problèmes d'espace, de distance, de repérage dans des lieux chargés d'altérité. D’une part, comme l'écrit Jacques Lévy (2013) « La compétence peut se nourrir des expériences accumulées dans ces espaces ["ceux dans lesquels il a accumulé une compétence d'usage»] mais elle ouvre la possibilité d'économiser du temps, de l'énergie, des épreuves dans de nouveaux espaces », ainsi les individus qui ont acquis la maîtrise du métro dans leur quotidien ont la capacité de la réinvestir dans d'autres villes. Mais d'autre part, les réseaux métropolitains ne sont pas homogènes. Des variations dans les conceptions, mais aussi comme nous l'avons vu des perturbations inattendues surviennent. Et l'usage du métro suppose aussi l'appropriation d'un système de signes et d'informations qui doit être médié soit dans une perspective touristique rendue accessible à des individus confrontés à une forte altérité. Ces médiations sont portées par des acteurs qui souhaitent mettre à la disposition des individus des moyens qualifiés de prise ou d'affordance qui atténuent localement l'altérité. Ces dispositifs sont conçus en s'appuyant sur des dispositions supposées acquises, mais nécessitent leur mobilisation en compétences par les touristes. Cela crée au sein des espaces marqués par une forte altérité des îlots de familiarité différenciée ou d'altérité partielle qui peuvent prendre la forme de réseaux si les prises s'inscrivent dans une conception et un traitement globaux de l'espace fréquenté par les touristes. Ainsi nous avons observé que, dans le centre de Saint-Pétersbourg, la signalétique urbaine en surface prend le relais des prises installées dans le métro et permet d'accéder de manière autonome aux établissements touristiques. Au contraire à Shanghai, les individus sont davantage démunis dès qu'ils quittent le réseau métropolitain. Mais dans la ville russe, la rupture intervient dès que l'on souhaite accéder aux sites touristiques situés en périphérie, notamment les palais Peterhof ou Tsarskoïe Selo situés à $25 \mathrm{~km}$ du centre.

31 Cette approche pourrait être complétée par une analyse des politiques urbaines et touristiques menées dans ces villes afin de déterminer les freins opposés à la construction de la familiarité. Viennent-ils de la politique touristique, de ses insuffisances, de ses faiblesses relatives? Est-ce la faiblesse de la fréquentation qui n'incite pas à se lancer dans des arrangements coûteux, la rentabilité économique n'étant pas assurée ? Sont-ils plutôt le fait de la résistance des acteurs urbains des transports peu sensibilisés au tourisme? En effet, dans les villes historiquement constituées et investies par le tourisme, les enjeux posés par cette mobilité ne sont pas évidents, au contraire de stations ou villes spécialisées, créées par et pour le tourisme, portées par des acteurs informés (Violier, 2009). Par ailleurs la verticalité des institutions publiques induit des contradictions entre les acteurs du tourisme qui souhaiteraient des dispositifs de prise ou d'affordance et ceux de la voierie qui sont mus par d'autres logiques. 


\section{BIBLIOGRAPHIE}

Gilles Brougère, « Soi-même comme apprenant. Essai d'autoethnographie », dans Gilles Brougère et Giulio Fabiano (dir.), Apprentissages en situation touristique, «Éducation et didactiques », Presses universitaires du Septentrion, 2014.

Giorgia Ceriani, Vincent Coëffé, Jean-Christophe Gay et alii, « Conditions géographiques de l'individu contemporain », Espacestemps.net, « Textuel », 2008. En ligne [consulté le 2 mars 2017] : http://www.espacestemps.net/articles/conditions-geographiques-individu-contemporain/ Daniel Clary, Le Tourisme dans l'espace français, Masson, 1993.

Alain Corbin, Le Territoire du vide. L'Occident et le désir du rivage, 1750-1840, Flammarion, 1990.

Philippe Duhamel et Rémy Knafou, « Le tourisme dans la centralité parisienne ", dans Thérèse Saint-Julien et Renaud Le Goix (dir.), La Métropole parisienne. Centralités, inégalités, proximités, Belin, 2007.

Philippe Duhamel et Philippe Violier, Tourisme et littoral, Belin, 2009.

Émilie Flon, « De la mise en scène à la fiction touristique : la médiation du voyage urbain sur la ligne de métro Xinbeitou à Taipei », Espaces et sociétés, n 151, 2012, p. 85-101.

Jean-Christophe Gay, « Deux figures de retranchement touristique : l'île hôtel et la zone franche ", Mappemonde, $\mathrm{n}^{\circ}$ 59, 2000, p. 10-16.

James J. Gibson, The Ecological Approach to Visual Perception, Lawrence Eribaum Associates, 1986.

Samuel Huntington, The Clash of Civilizations and the Remaking of World Order, Simon \& Schuster, 1996.

Isaac Joseph, « Un monde sensible », Informations sociales, vol. 33, 1994.

Isaac Joseph, La Ville sans qualités, Éditions de l'Aube, 1998.

Rémy Knafou, « Scènes de plage dans la peinture hollandaise du XVII ${ }^{\mathrm{e}}$ siècle, l'entrée de la plage dans l'espace des citadins », Mappemonde, $\mathrm{n}^{\circ}$ 58, 2002.

Rémy Knafou et Mathis Stock, « Tourisme », dans Jacques Lévy et Michel Lussault, Dictionnaire de la géographie, Belin, 2003, p. 931-934.

Jacques Lévy, «Capital spatial », dans Jacques Lévy et Michel Lussault, Dictionnaire de la géographie , Belin, 2013, p. 124-126.

Michel Lussault, « Compétences spatiales », dans Jacques Lévy et Michel Lussault, Dictionnaire de la géographie, Belin, 2013, p. 212-214.

Équipe Mit, Tourismes. 1, Lieux communs, « Mappemonde », Belin, 2002.

Équipe Mit, Tourismes. 2, Moments de lieu, « Mappemonde », Belin, 2005.

Gwendal Simon, Pratiques touristiques dans la métropole parisienne : une analyse des mouvements intraurbains, thèse de sociologie, université de Paris-Est, 2010.

Mathis Stock, Mobilités géographiques et pratiques des lieux. Étude théorico-empirique à travers deux lieux touristiques anciennement constitués : Brighton \& Hove (Royaume-Uni) et Garmisch-Partenkirchen (Allemagne), thèse de géographie, université de Paris-7, 2001. 
Mathis Stock (dir.), Tourisme, acteurs, lieux, enjeux, Belin, 2003.

Mathis Stock, «Il Mondo è mobile », dans Jacques Lévy (dir.), L'Invention du Monde, Les presses de Sciences po, 2008.

Laurent Tissot, Naissance d'une industrie touristique, les Anglais et la Suisse au XIX ${ }^{e}$ siècle, Payot, 2000.

Philippe Violier, « Proposition pour un modèle d'analyse de la dynamique des lieux touristiques », dans Jean-Pierre Lemasson et Philippe Violier (dir.), Destinations et territoires, t. 1 : Coprésence à l'œuvre, Téoros, Presses universitaires du Québec, 2009, p. 26-37.

\section{NOTES}

1. «Chiffres-clé du tourisme » 2015, publication de la Direction générale de la compétitivité de l'industrie et des services.

2. Eurostat 2006 [consulté le 31 novembre 2016] : ec.europa.eu

3. Nous relevons par exemple l'apparition depuis 2009 d'un opérateur d'un nouveau genre, la société Evaneos, ni tour-opérateur puisqu'il n'élabore pas de circuits en propre, ni agence de voyage car il ne diffuse pas des circuits réalisés par des tour-opérateurs, il propose en revanche une médiation avec des agences réceptives qu'il sélectionne et auprès desquelles il perçoit une commission.

4. Par exemple, retarder un retour permettant de réaliser des économies...

5. Le flou de la définition du tourisme utilisée par l'Organisation mondiale du tourisme nous incite à la prudence et à considérer pour ces statistiques les voyageurs et non les touristes.

\section{RÉSUMÉS}

Partant du constat que la mobilité des individus se heurte à l'altérité des mondes, trois démarches sont mobilisées pour comprendre comment la circulation est possible : la première met en valeur le capital spatial des individus, la seconde s'intéresse aux technologies spatiales qui la facilitent, la troisième, dans laquelle le présent texte s'intègre, pose la question de la familiarité construite, soit des arrangements instaurés par des acteurs pour atténuer ponctuellement la contrainte de la diversité que le monde oppose à l'habiter mobile. Ces bricolages constituent des prises grâce auxquelles l'individu peut affronter l'altérité de manière autonome. Une fois développés les attendus théoriques, l'article développe une approche pragmatique fondée sur l'observation des réseaux de métro dans des métropoles situées dans différents mondes (New York, Berlin, Saint-Pétersbourg, Moscou, Shanghai). Il en résulte que le différentiel d'altérité perçu ne correspond pas à l'altérité objective. Alors que cette dernière situe les villes selon un continuum d'altérité croissante dans l'ordre de citation de ces villes, soit NewYork et Berlin / Saint-Pétersbourg / Moscou / Shanghai, l'altérité perçue par un touriste occidental utilisant le métro sera plutôt New-York et Berlin / Shanghai / Saint-Pétersbourg / Moscou. De nombreux arrangements rendent le métro de la métropole chinoise plus facile d'usage que celui des métropoles russes et surtout de celui de Moscou. 
Runner of the report that the mobility of the individuals comes up against the otherness of the worlds, three steps initiatives are mobilized for understanding how the circulation is possible: the first one emphasizes the spatial capital of the individuals, the second is interested in the spatial technologies which facilitate it, the third, into which the present text becomes integrated, asks the question of the constructed familiarity, or arrangements established by actors to limit punctually the constraint of the diversity which the world brings into conflict with mobility. These tinkering constitute affordances which the individual can face the otherness in an autonomous way. Once presses the theoretical reasons adduced, the article develops a pragmatic approach based on the observation of metro networks in metropolises situated in various worlds (New York, Berlin, Saint-Petersburg, Moscow, Shanghai). As a result the received difference of otherness does not correspond to the objective otherness. While the latter places cities according to a continuum of increasing otherness in the order of quotation of these cities, that is New York and Berlin / Saint-Petersburg / Moscow / Shanghai, the otherness perceived by a western tourist using the subway will be rather New York and Berlin / Shanghai / Saint-Petersburg / Moscow. Numerous arrangements return the subway of the easier Chinese metropolis of use than that of the Russian metropolises and especially that of Moscow.

INDEX

Mots-clés : technologies spatiales, prise, affordance, médiation, altérité

Keywords : spatial technology, affordance, mediation, otherness

\section{AUTEUR}

\section{PHILIPPE VIOLIER}

Professeur de géographie, directeur de l'UFR Esthua Tourisme et Culture, université d'Angers philippe.violier@univ-angers.fr 